\title{
Field Coil Design
}

\author{
Yilmaz İsPí** \\ Fen Fakultesi, Jeofizik, Istanbul \\ (Received April 25, 1969 Revised May 7. 1969)
}

\begin{abstract}
This paper is specifically aimed at the problem of obtaining magnetic fields of the highest possible uniformity at the center of coil systems of a number of given shapes, principally combinations of circular, square and solenoidal coil. The field uniformities of some previous arrangements and some new arrangements are discussed. The variations of the onaxis fields due to different coil arrangements are shown graphically (Fig. 3 and 10) near their centers and tabulated numerically along their axes of symmetry.
\end{abstract}

\section{Introduction.}

It is often necessay to obtain a field with a certain uniformity in magnitude and in direction over a required region in which a sample or an apparatus is being placed. In addition, this region of high uniformity must be as large as possible compared with the volume of the space of the coil system producing the field. However, in ordinary applications, large coil systems have been used because of their open construction.

Ruark and Peters (1926), Beyerle (1931), Harris (1934a, b) and Lyddons and Ruark (1939) have designed field coil arrangements which consist of a pair or circular or rectangular coils giving a progressively better uniformity near the center and along the symmetry axis.

A system of five, equally-spaced, square coils contained in a cubic envelope was first suggested by Rubens (1945). Parry (1967) has arranged a pair of square coils under the Helmholtz condition, that is, all second order terms disappear. He has also given three useful diagrams to show the relations between field derivatives for coil systems with tetragonal and cylindrical symmetries.

Two pairs of circular coils have been used by Fanselau (1929), and Thellier $(1938,1967)$ to obtain a uniform field along only one direction.

In the present study some new coil arrangements and the possibility of obtaining improved uniformity from previous systems are investigated. All calculated field values are normalized to the value at the geometrical center. Also, the radii of the circular coils and solenoids and the half-length of one edge of the square coils are often taken as unit length to facilitate comparison and to make the designs of the coil systems easily adoptable to different dimensions.

Calculations of the field variations along the symmetry axes are made by computer (IBM 360).

* Now at The School of Physics, The University, Newcastle upon Tyne 1, U.K. 


\section{Uniformity of Field Due to Circular and Square Coils.}

The axial magnetic field component of both circular and square field coils may be formulated as Taylor series in terms of $z$, near the center,

$$
H_{z}(z)=H_{z}(0)+b_{2} z^{2}+b_{4} z^{4}+\cdots \cdots+b_{n} z^{n}
$$

On the other hand, the field components parallel to the symmetry axis and along the radius for a small volume in the center are expressed in $z$ and $r$ in the case of cylindrical symmetry (Parry 1967).

$$
\begin{aligned}
& H_{z}(z, r)=H_{z}(0,0)+b_{2}\left(z^{2}-\frac{r^{2}}{2}\right)+b_{4}\left(z^{4}-3 z^{2} r^{2}+\frac{3}{8} r^{4}\right)+\cdots \cdots \\
& H_{r}(z, r)=-b_{2} z r+b_{4}\left(\frac{3}{2} z r^{3}-2 z^{3} r\right)+\cdots \cdots
\end{aligned}
$$

where

$$
b_{n:}=\frac{1}{n !}\left(\frac{\partial^{n} H_{z}}{\partial z^{n}}\right)_{0}
$$

The expansions of the components of the magnetic field near the center in cartesian co-ordinates for the case tetragonal symmetry are

$$
\begin{aligned}
& H_{z}(z, r)=H_{z}(0,0)+b_{2}\left(z^{2}-\frac{r^{2}}{2}\right)+b_{4}\left(z^{4}-3 z^{2} r^{2}+3 x^{2} y^{2}\right)+c_{4}\left(r^{4}-8 x^{2} y^{2}\right)+\cdots \cdots \\
& H_{x}(z, r)=-b_{2} z x+b_{4}\left(-2 z^{3} x+6 x y^{2} z\right)+c_{4}\left(8 z x^{3}-12 x y^{2} z\right)+\cdots \cdots \\
& H_{y}(z, r)=-b_{2} z y+b_{4}\left(-2 z^{3} y+6 y x^{2} z\right)+d_{4}\left(8 z y^{3}-12 y x^{2} z\right)+\cdots \cdots
\end{aligned}
$$

where

$$
\begin{aligned}
& c_{n}=\frac{1}{n !}\left(\frac{\partial^{n} H_{z}}{\partial x^{n}}\right)_{0}, \quad d_{n}=\frac{1}{n !}\left(\frac{\partial^{n} H_{z}}{\partial y^{n}}\right)_{0} \\
& r=\left(x^{2}+y^{2}\right)^{1 / 2}
\end{aligned}
$$

The uniformities in magnitude of the field due to both types of coil, near the centers depend on the difference of $H_{z}(z, r)-H_{z}(0,0)$ and the value of $H_{r}(z, r)$. In other words, the values of the coefficients, $b_{n}$, produce perturbations in the magnetic field near the center. The field uniformities in direction depend on the ratios of $H_{r}(z, r)$ to the difference of $H_{z}(z, r)-H_{z}(0,0)$ in both cases.

To reach the absolute uniformity in the central region of a coil system with cylindrical or tetragonal symmetry, successive derivatives of the field components must be zero, as is well known. In the simplest case, it is possible to obtain the first approximation when $b_{2}=0$ for the field uniformity in both arrangements which are known as the Helmoltz and Parry pairs. To approach the second approximation, when all second and fourth derivatives vanish, it will be necessary to take a combination of more than two coils. 


\section{Circular Coil and Helmholtz Pair}

Generally, there are two different arrangements of the Helmholtz pair as is known. One of them is called the Helmholtz-Gaugain system where the appropriate separation of a pair of circular coils is equal to the radius of the coil, i.e. $\theta=63^{\circ} 26^{\prime} .2$. This case is known as "the Helmholtz Condition" or "the first approximation". The second arrangement has a separation that is about 1.2 times the radius, $i . e . \theta \cong 59^{\circ}$. It is used to obtain the same value of the onaxis field at $0, A$ and $B$ points which are shown in Fig. 1 (Bouasse 1914, Fanselau 1929).

Here the first system is discussed because the aim is to obtain a uniform magnetic field near the center.

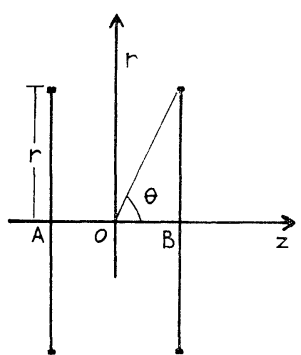

Fig. 1 The radial section of the Helmholtz Pair.

The expressions for the axial magnetic field and its derivatives, due to a circular coil,

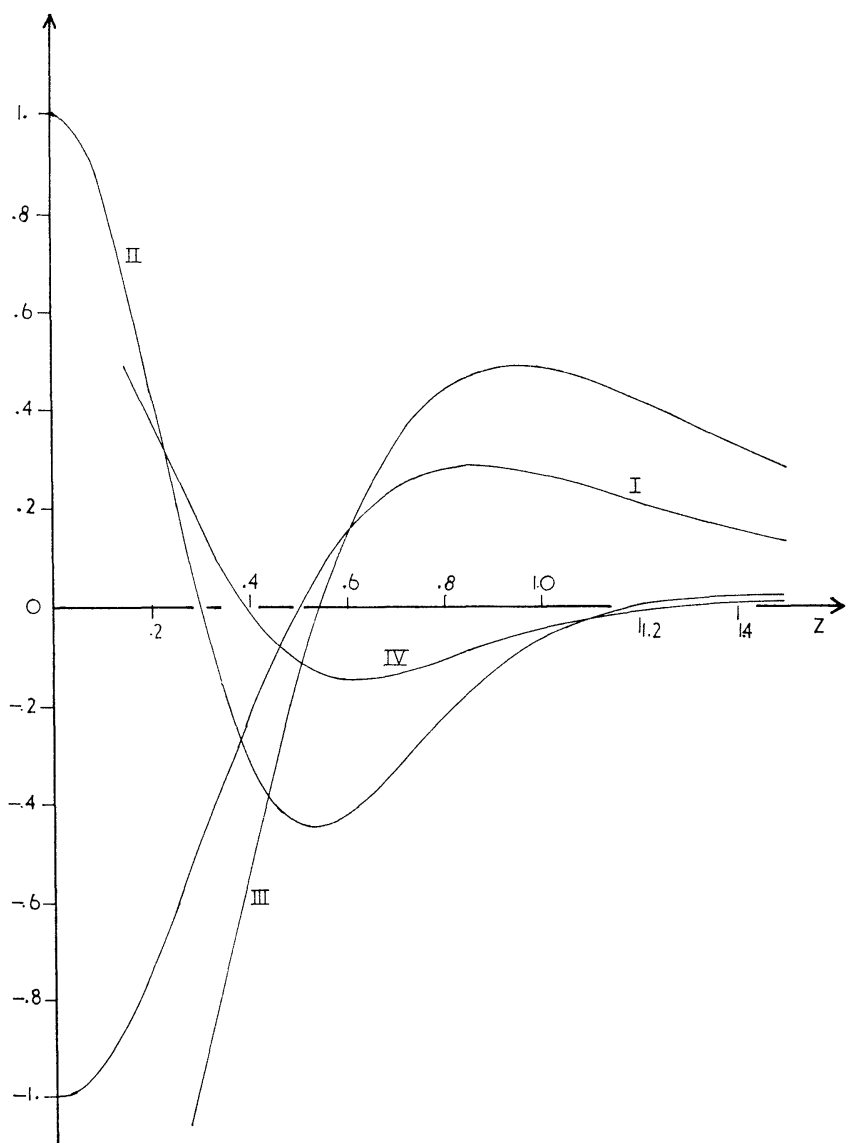

Fig. 2 Axial derivatives of the magnetic field due to a single coil. I and II represent the second $\left(\mathrm{H}_{z}{ }^{\prime \prime} / 3 \mathrm{c}\right)$ and the fourth $\left(\mathrm{H}_{z}{ }^{i v} / 45 \mathrm{c}\right)$ derivatives due to a circular coil; III and IV represent the second $\left(\mathrm{H}_{z}{ }^{\prime \prime} / \mathrm{c}_{1}\right)$ and the fourth $\left(\mathrm{H}_{z}{ }^{i v} / \mathrm{c}_{1}\right)$ derivatives due to a square coil. 
with respect to $z$, are

$$
\begin{aligned}
& \frac{H_{z}}{c}=\left(1+z^{2}\right)^{-3 / 2} \\
& \frac{H_{z}^{\mathrm{II}}}{3 c}=\frac{1-4 z^{2}}{\left(1+z^{2}\right)^{7 / 2}} \\
& \frac{H_{z}^{\mathrm{IV}}}{45 c}=\frac{1-12 z^{2}+8 z^{4}}{\left(1+z^{2}\right)^{11 / 2}}
\end{aligned}
$$

where $c=2 \pi N i$ and $r=1$

$N$ is the number of turns.

In this case, the second derivative vanishes at $z=0.50$ as is well known. But the value of the fourth derivative vanishes at only $z=0.30$ and $z=1.17$ (Fig. 2, curves I and II).

The variations of the field components, $H_{z}$ and $H_{r}$, along the $0 z$ and $0 r$ axes due to the Helmholtz pair are shown as curves I (Fig. 3), II and III (Fig. 4). The offaxis field due to this arrangement is calculated using the available tables (Hart, 1967). Also the change of angle between the direction of the total magnetic field and $0 z$ axis, is plotted along $0 r$ axis (Fig. 5, curve I). The percentage variations in magnitude and in direction are also marked on the curves: $(+)$ and $(-)$ indicate that the value is greater and less, respectively, than the value at the centre (Fig. 3, 4 and 5).

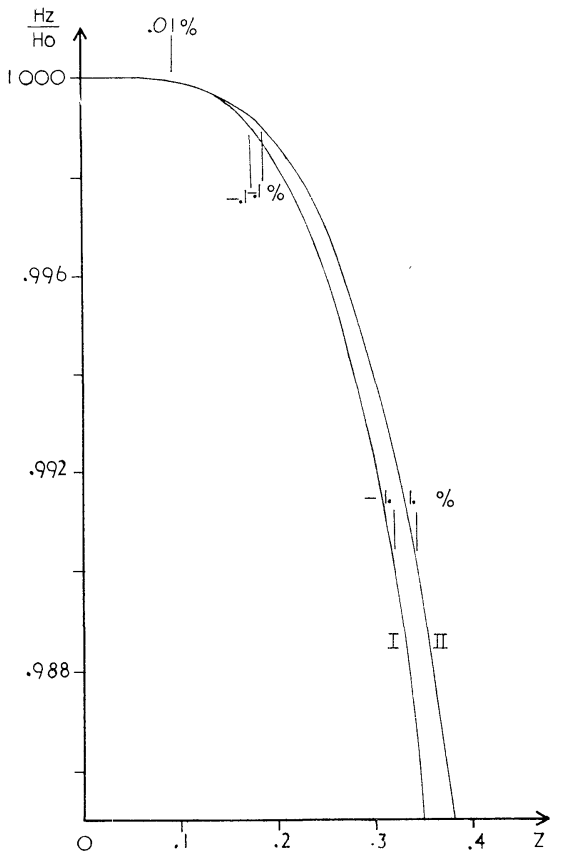

Fig. 3 The axial variations due to both the Helmholtz pair (I) and the Parry pair (II).

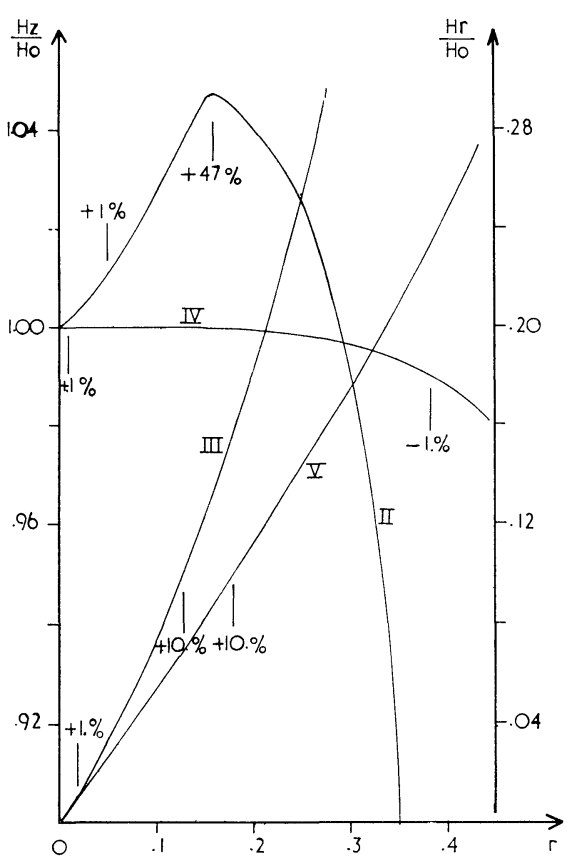

Fig. 4 The radial variations of the components of the fields due to both the Helmholtz pair (II, III) and the Parry pair (IV, V). Curves II and IV show the axial conponents ; III and V show the radial components. 


\section{Square Coil and Parry Pair}

The magnetic field due to a square coil along its symmetry axis can be expressed as Parry (1967),

$$
\frac{H_{z}}{c_{1}}=\frac{1}{\left(1+z^{2}\right)\left(2+z^{2}\right)^{1 / 2}} \quad \text { for } \quad \mathrm{a}=1, c_{1}=8 \mathrm{ni}
$$

where $2 \mathrm{a}$ is the length of one edge of the square coil. The expressions for the field derivatives can be given as follows

$$
\begin{aligned}
& \frac{H_{z}^{\mathrm{II}}}{c_{1}}=H_{z}^{\mathrm{I}} X+H_{z} X^{\mathrm{I}} \\
& \frac{H_{z}^{\mathrm{IV}}}{c_{1}}=H_{z}^{\mathrm{III}} X+3 H_{z}{ }^{\mathrm{II}} X^{\mathrm{I}}+3 H_{z}{ }^{\mathrm{I}} X^{\mathrm{II}}+H_{z} X^{\mathrm{III}}
\end{aligned}
$$

with

$$
X=z\left(\frac{2}{1+z^{2}}+\frac{1}{2+z^{2}}\right)
$$

The second axial derivative vanishes where $z_{0}=0.5441$, but the fourth derivative vanishes at different distances, i. e. $z_{1}=0.390$, and $z_{2}=1.280$ (Fig. $6 \& 2$-III, -IV). The distance between two square coils must be taken as 1.0882 with respect to the Helmholtz condition, so that all second order terms disappear (Parry 1967).

The ratios of $H_{z} / H_{0}$ and $H_{r} / H_{0}$ due to the single square coil with $2 \mathrm{a}=1$, at any point on the $(x, z)$ plane are expressed as,

$$
\begin{aligned}
\frac{H_{z}(x, z)}{H\left(0, z_{0}\right)}= & {\left[\frac{1-x}{A}\left(\frac{1}{D}+\frac{1}{1+z^{2}}\right)+\frac{1+x}{B}\left(\frac{1}{C}+\frac{1}{1+z^{2}}\right)\right] / \frac{4}{\sqrt{2+z_{0}^{2}\left(1+z_{0}^{2}\right)}} } \\
& \left.\frac{H_{r}(x, z)}{H\left(0, z_{0}\right)}=z\left(\frac{1}{D . A}-\frac{1}{C . B}\right)\right) \frac{4}{\sqrt{2+z_{0}^{2}\left(1+z_{0}^{2}\right)}}
\end{aligned}
$$

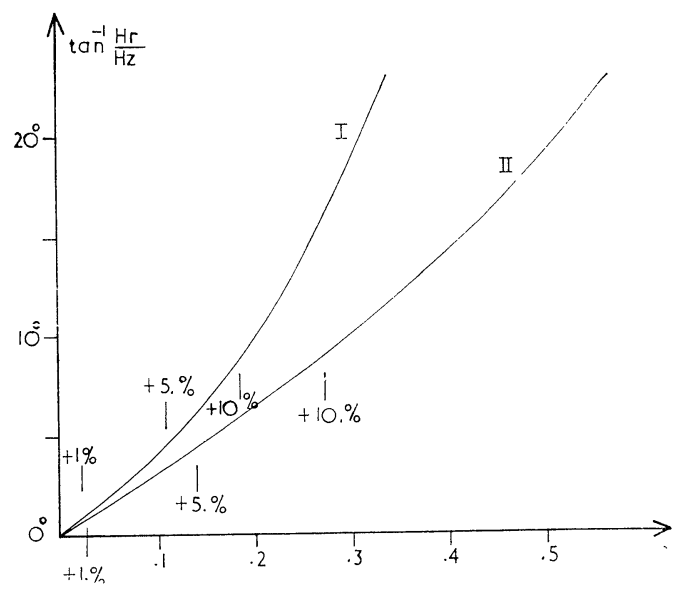

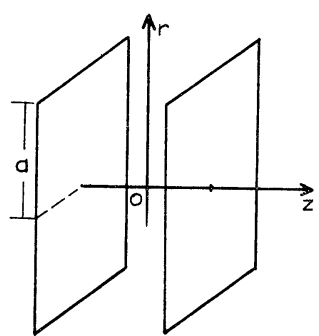

Fig. 6 The Parry pair.

Fig. 5 The angular variations of the total magnetic fields due to both the Helmholtz pair (I) and the Parry Pair (II) in radial direction. 
with

$$
\begin{array}{ll}
A=\left[(1-x)^{2}+1+z^{2}\right]^{1 / 2}, & B=\left[(1+x)^{2}+1+z^{2}\right]^{1 / 2} \\
C=(1+x)^{2}+z^{2}, & D=(1-x)^{2}+z^{2}
\end{array}
$$

where

$H\left(0, z_{0}\right)$ is the value of the magnetic field at the the center produced by the pair where $z_{0}=0.5441$.

$H_{z}$ and $H_{r}$ are the values of the $0 z$ and $0 r$ components of the offaxis field due to one coil at any point on $(x, z)$ plane.

The functions of $H_{z}(0, z) / H_{0}, H_{z}(x, 0) / H_{0}, H_{r}(x, 0) / H_{0}$ and $\tan ^{-1}\left(H_{r}(x, 0) / H_{z}(x, 0)\right)$ due to the Parry pair are plotted along the $0 z$ and $0 r$ axes as curves II (Fig. 3); IV, V (Fig. 4), and II (Fig. 5), respectively.

\section{Solenoid and Solenoid Pair with a Gap}

The onaxis magnetic field due to a finite solenoid, if the measuring point is outside of the solenoid, can be expressed as (Fig. 7),

$$
H_{z}=-2 \pi n_{1} i\left(t_{1}-t_{2}\right)
$$

where $n_{1}$ is the number of turns in each unit length along the symmetry axis,

$$
\begin{aligned}
& t_{1}=\cos \theta_{1}=\frac{B-z}{\left[(B-z)^{2}+1\right]^{1 / 2}} \\
& t_{2}=\cos \theta_{2}=\frac{e-z}{\left[(e-z)^{2}+1\right]^{1 / 2}}
\end{aligned}
$$

for $r=1$, and $B=e+A$

The derivatives of the function $t_{1}$, with respect to $z$, at the point 0 , are (Fig. 7)

$$
\begin{aligned}
& t_{1}{ }^{\mathrm{II}}=-\frac{3 B}{\left(B^{2}+1\right)^{5 / 2}} \\
& t_{1}{ }^{\mathrm{IV}} / 15=\frac{3 B-4 B^{3}}{\left(B^{2}+1\right)^{9 / 2}}
\end{aligned}
$$

The expressions for the derivatives of $t_{2}$ are of course exactly similar to those for the derivatives of $t_{1}$ but with $B$ and $e$ interchanged.

For the first approximation of the field uniformity of two equi-dimensional axial solenoids we have a lot of solutions: One of them will give the arrangement of the Helmholtz pair as

$$
H_{z}{ }^{\mathrm{II}}=0 \text { for } A=0 \text { and } B=e=0.5
$$

Fig. 8 shows the functions of the second and fourth derivatives of $t_{1}$ with respect to $z$ along $0 z$ axis. For a certain value of $A$, the second deriva-

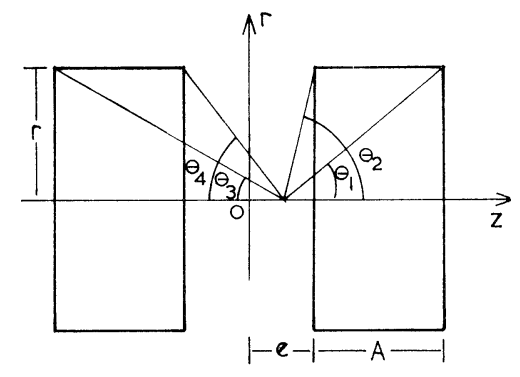

Fig. 7 The radial section of two coaxial solenoids. 


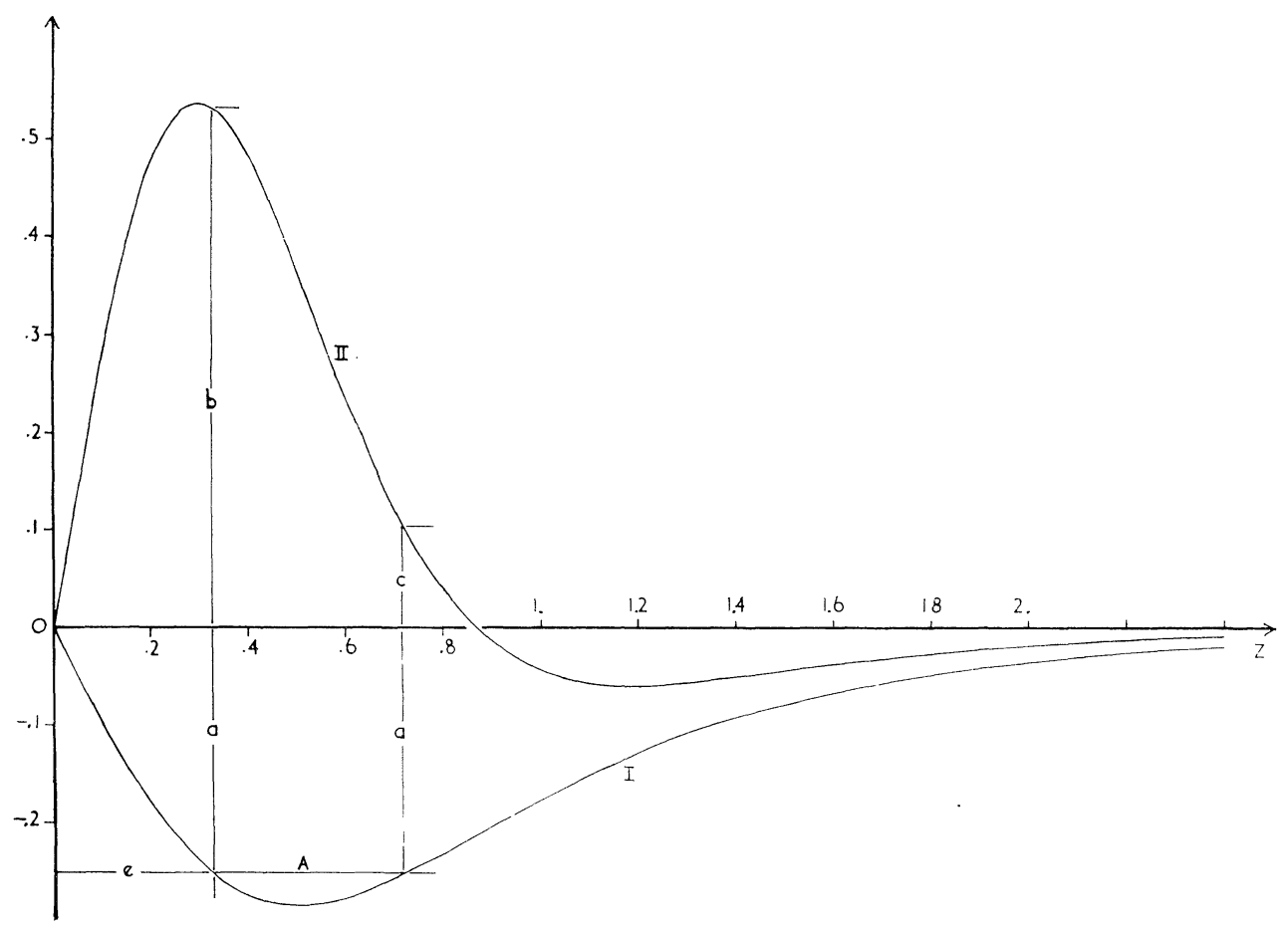

Fig. 8 The second (I) and the fourth (II) derivatives of the function $t_{1}$.

tive of the field produced by the pair is zero, i.e. $(-a-(-a))=0$. The value of the fourth derivative is proportional to the difference $(b-( \pm c))$. Therefore, four different values of $A$, the variations of the half-length of the gap (I) and the value of the fourth derivative (II) can be determined (Fig. 9), and convenient dimensions can be chosen for any required system with the aid of Fig. 8 and 9.

The ratio of the onaxis field-to-the field at the centre, 0 , can be given for two equidimensional and coaxial solenoids with a gap as,

$$
\frac{H_{z}}{H_{0}}=\frac{\cos \theta_{1}-\cos \theta_{2}+\cos \theta_{3}-\cos \theta_{4}}{2\left(\cos \theta_{0}-\cos \theta_{0}{ }^{1}\right)}
$$

where $\theta_{0}$ and $\theta_{0}{ }^{1}$ are the values of $\theta_{1}$ (or $\theta_{3}$ ) and $\theta_{2}$ (or $\theta_{4}$ ) at the center of this system (Fig. 7).

Fig. 10 (curve $\mathrm{I}$ ) shows the variation of the ratio $\mathrm{H}_{z}$ over $\mathrm{H}_{0}$ or along the $0 z$ axis in the case where $B=1.0, e=0.194, A=0.806$ for $r=1.0$.

\section{Thellier's Systems}

The first of two systems used by Thellier $(1938,1967)$ consists of four coaxial circular coils at equal distances (Fig. 11). The perturbations in the fields due to this system and due to the central pair only are shown in Fig. 10 (curve II) and Fig. 3 (curve I), respectively, in the same scale. The radii of all coils are taken as unit length in calculation.

The other arrangement (Thellier 1938, 1967) involves two Helmholtz pairs where the ratio of the radii of the outside pair to the radii of the central pair has been taken as 2 


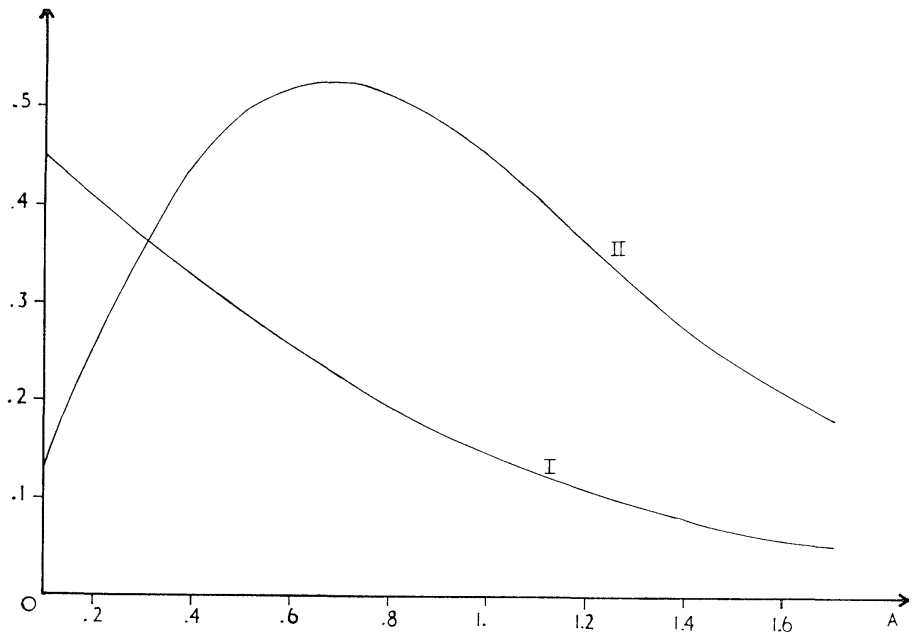

Fig. 9 Variations of the half-width of the gap (I), and the value of the fourth derivative (in terms $t_{1}^{i v}$ ) depend on the length of the solenoid (A) when the second derivative vanishes.

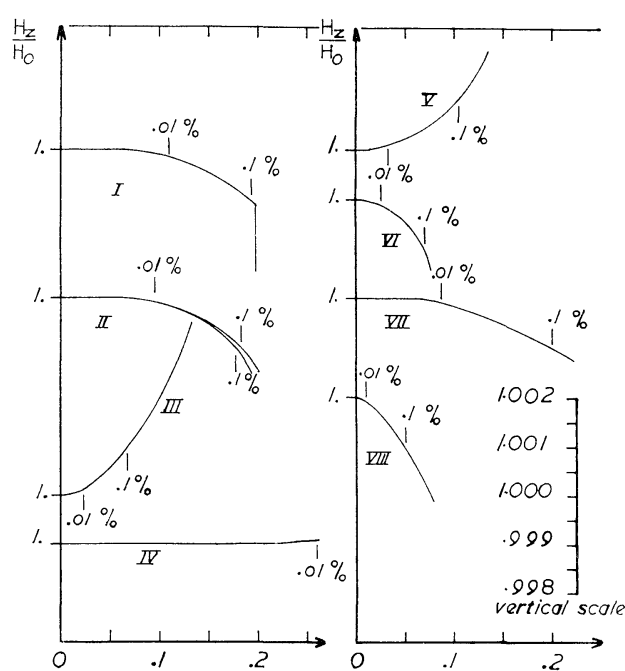

Fig. 10 Variations of the onaxis fields produced by all the arrangements.

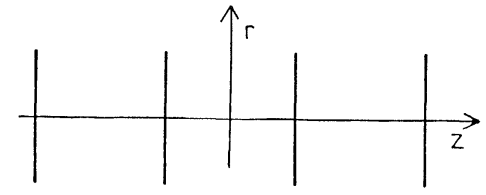

Fig. 11 The radial section of the Thellier system, I.

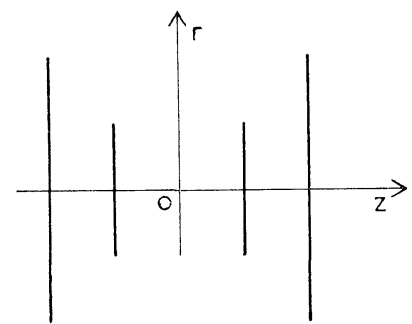

Fig. 12 The radial section of the Thellier system, II:

(Fig. 12). The ratio of the number of turns of the central pair to the others suggested by Thellier is 4, and in Fig. 10 turns ratios of 4 and 2 are compared (curves III-a, -b). In this calculation, the radius of the central pair is taken as unit length.

\section{Fanselau's System}

The dimensions of three field coil arrangements have been determined by Fanselau (1929). They consist of one (i.e. Helmholtz coil system), two and three circular pairs, respectively, giving a progressively better uniformity near the centre and along the symmetry axis. 
The second of them is shown in Fig. 13, as coaxial circular coils where the distances from the geometrical center of the system to the center of an outside and a central coil are 0.90673 and 0.29757 , respectively, and the radii of the outside and the central pairs are taken as 0.76317 and 1.0. Each of the coils has the same number of turns.

Fig. 10 (curve IV) shows the perturbations in the onaxis field produced by this system with the above dimensions.

\section{Tow Pairs of Square Coils}

This system involves two coaxial square coil pairs. The ratio of the radii (central over outside) is 2.0. The distances between the geometrical centre and the centres of the central and the outside coils are taken as 0.30 and 0.7014 (Fig. 14). These dimensions are determined using Fig. 2 (curves III and IV), in order to approach the second approximation in the uniformity as near as possible.

The variations of the onaxis field produced by this system are shown in Fig. 10 (curve V) with the same dimensions, and each coil has the same number of turns.

\section{Development of Lavaud's System}

Lavaud (1964) has designed a field coil system which consists a finite solenoid with a circular coil pair added to the extremes of it to obtain the first approximation in uniformity.

In the present study it is shown that the second approximation in the uniformity can be obtained with the aid of a circular pair which has different dimensions (Fig. 15).

The second and fourth derivatives of the axial field due to a finite solenoid and a circular coil pair are respectively as follows ( $\dot{\mathrm{I}}_{\text {spir, 1966), }}$

$$
\begin{aligned}
& H_{z}^{\mathrm{II}}=6 \pi n_{1} i\left(-\frac{2 l r^{2}}{\left(r^{2}+l^{2}\right)^{5 / 2}}\right) \\
& H_{z}^{\mathrm{IV}}=60 \pi n_{1} i\left(\frac{3 l r^{4}-4 l^{3} r^{2}}{\left(r^{2}+l^{2}\right)^{9 / 2}}\right) \\
& \mathscr{H}_{z} \mathrm{II}=12 \pi N i\left(\frac{R^{2}\left(4 L^{2}-R^{2}\right)}{\left(R^{2}+L^{2}\right)^{7 / 2}}\right) \\
& \mathscr{H}_{z}{ }^{\mathrm{IV}}=60 \pi N i\left(\frac{R^{2}\left(R^{2}-12 R^{2} L^{2}+8 L^{4}\right)}{\left(R^{2}+L^{2}\right)^{11 / 2}}\right)
\end{aligned}
$$

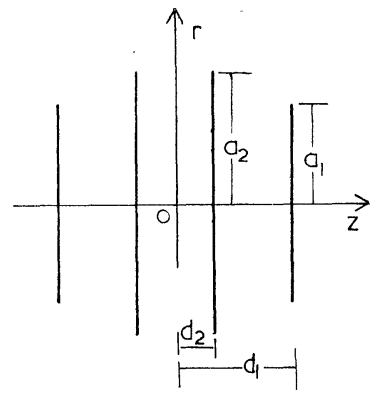

Fig. 13 The radial section of the Fanselau system.

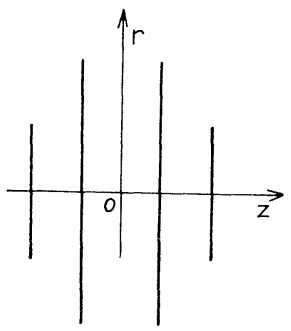

Fig. 14 The section of two pairs of square coils.

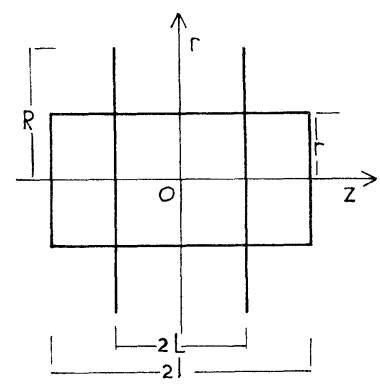

Fig. 15 The radial section of the development of the Lavand system. 
where the dimensions, $l, r, R$ and $L$ are shown in Fig. 15.

The sums of $\left(H_{z}^{\mathrm{II}}+\mathscr{H}_{z}^{\mathrm{II}}\right)$ and $\left(H_{z}^{\mathrm{IV}}+\mathscr{H}_{z}^{\mathrm{IV}}\right)$ are zero under the following conditions, respectively

$$
\begin{aligned}
& n_{1}=-\frac{r^{2}\left(4 v^{2}-1\right)\left(u^{2}+1\right)^{5 / 2}}{R^{3} u\left(1+v^{2}\right)^{7 / 2}} \cdot N \\
& n_{1}=-\frac{r^{4}\left(1-12 v^{2}+8 v^{4}\right)\left(1+u^{2}\right)^{9 / 2}}{R^{5} u\left(1+v^{2}\right)^{11 / 2} \cdot\left(4 u^{2}-3\right)} \cdot N
\end{aligned}
$$

where

$$
u=\frac{l}{r}, \quad v=\frac{L}{R}
$$

The relations between the dimensions of the system are obtained from the solutions of the above expressions as,

$$
v=\sqrt{.1435}, \quad R=2 r \text { if } u=1 .
$$

or

$$
\begin{aligned}
& L=\sqrt{.1435} . R=\sqrt{.1435} .2 r=\sqrt{.1435} \cdot 2 l \\
& n_{1}=.1884 . N
\end{aligned}
$$

where

$n_{1}$ is the number of turns per unit length of solenoid

$N$ is the number of turns of each circular coil

Curve VI (Fig. 10) shows the variation of the onaxis field due to this arrangement with $R=2, r=1, l=1, L=0.7576, n_{1}=1.9$ and $N=10.1$.

\section{Four Solenoids with Gaps}

This is the development of the system of "two solenoids with a gap" adding a pair of solenoids at the extremes (Fig. 16).

In this case, to decrease the perturbations in the axial field the curves of the derivatives of the field due to a finite solenoid can be used again (Fig. 8). The initial length of $A$ is divided into two portions as $l_{1}$ and $l_{2}$ with a gap, $e_{2}$, under the condition that the sum of the values of the second derivative must be zero. Using this rule, a convenient shape for this solenoid arrangement can be determined and the perturbations due to the fourth derivative of the field can also be decreased to zero. The changes of $l_{1}, l_{2}, e_{2}$ and the fourth derivative (d) are given in Fig. 17 as a function of $e_{1}$.

The axial field of the augmented system is shown in Fig. 10 (curve VII), for the values of $l_{1}=.076, l_{2}=.134, e_{1}=.26, e_{2}=.37$ and $r=1$.

\section{General Comments}

The results are tabulated in two groups. In each group the value of the axial distance 


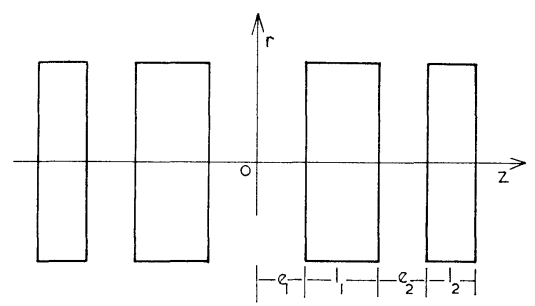

Fig. 16 The radial section of four solenoids.

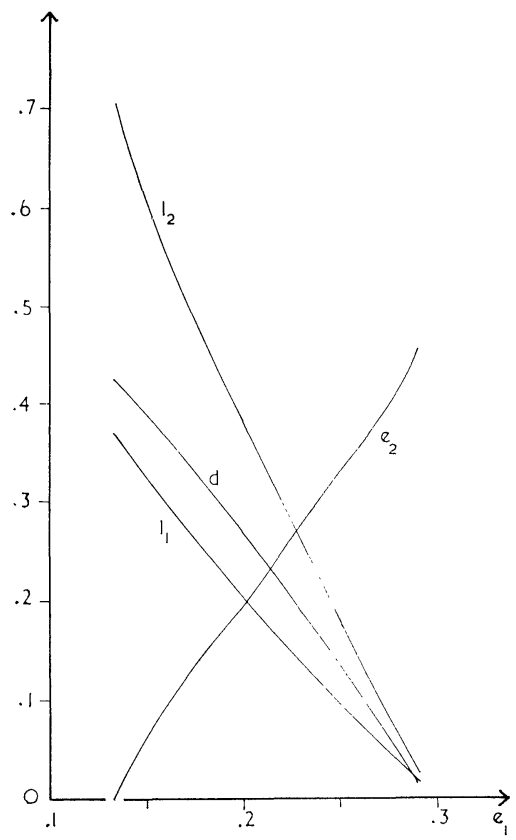

Fig. 17 The changes of $l_{1}, l_{2}, e_{2}$, and the fourth derivative (d) as a function of the half-width of the central gap (e) when the second derivative vanishes.

from the center of each arrangement is given for various degrees of uniformity.

It the first group values of these distances are calculated in terms of the radius or the half-width of the edge of each system. The dimensions have been given for each arrangement in the previous sections of this paper.

The second group of values of the distances are given at the right hand side of the table. These are calculated in terms of the distances from the centre of the arrangements to the inside edges of the first coil whether it is a circular, a square coil or a solenoid. These distances can be called the half-widths of the gaps. Only the results of the modified Lavaud's arrangement are presented in terms of the half-length of solezoid.

The arrangement can be separated into three groups according to their experimental applications, so a practicable system in each group may be chosen with the aid of Table 1-a and $-b$ giving the required uniformity-volume characteristics and ease of construction.

I. In practice, one, two or three Helmholtz or Parry pairs would usually be used for cancelling the total ambient field or any component. In all cases the Parry pair will probably be preferred to the Helmholtz pair as giving a higher uniformity in magnitude and indirection at the same region enclosing the centre (Fig. 3, 4 and 5). In addition, the construction of the Parry pair is easier, and a sample or an apparatus can be placed more accurately at the center.

II. In the second group of arrangements which are combinations of two coaxial pairs of 
Table 1 Amount of the Field Variations of the Systems

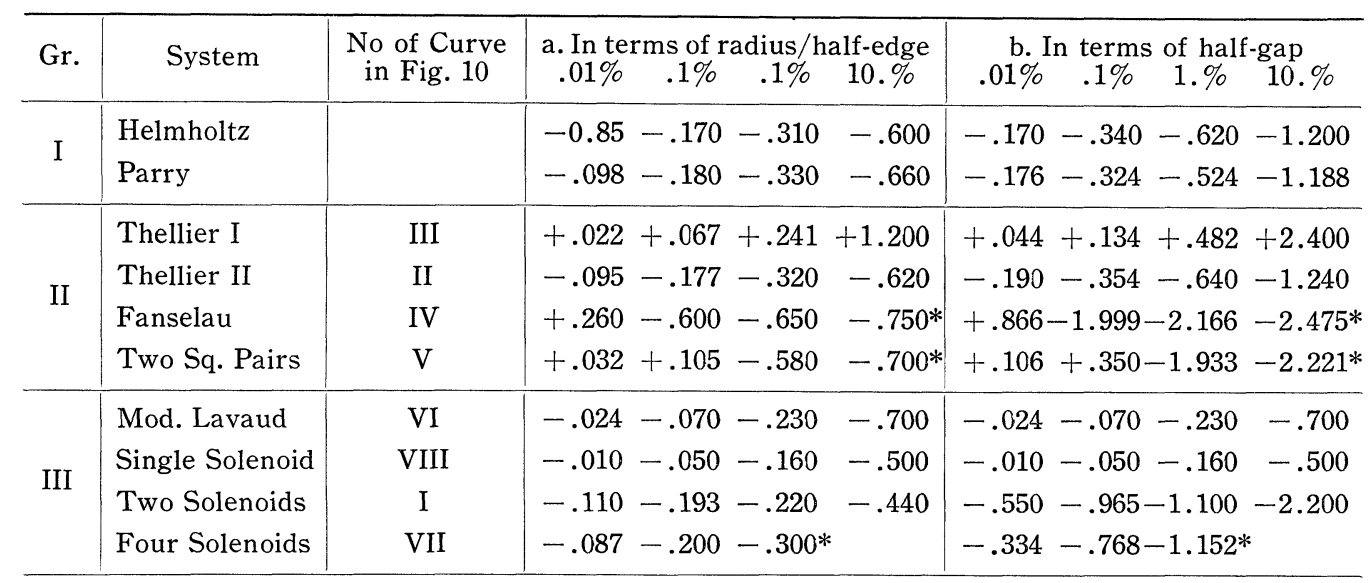

$(+)$ and $(-)$ indicate that the signs of the changes of the field value is greater and less, respectively, than the value of the geometrical centre of an arrangment.

* indicates that the normalized distance is greater than the number in the table.

circular or square coils. The best uniformity is produced by the Fanselau arrangement. The new system, "two pairs of square coils", will probably be preferred in most cases because its construction is easier and also the radial perturbations in the field due to a square pair are lower than the radial perturbations in the field due to a circular pair as is shown in Fig. 4. The uniformity of the field due to both Thellier systems with the outside pair is less than the uniformity obtained with the aid of only the Helmholtz or Parry pair, near the centre.

III. The last group involves two closed and two open arrangements of solenoids. The field uniformities due to both the "two solenoids" and "four solenoids" are higher than the field uniformities produced by "a single solenoid" with the length 2 units and 1 unit length radius (Table 1-a and-b; Fig. 10, curve VIII) or "modified Lavaud system". Therefore, these arrangements can be suggested instead of the closed construction, e.g. a finite solenoid, to obtain a magnetic field with a higher uniformity.

It should not be forgotten that the perturbations in the radial component of the field due to any coil system are always greater than the perturbations in the axial component.

The author acknowledges with pleasure the support and encouragement of Professor I. Özdogan. He gives his sincere thanks to Professor G. Grenet who introduced him to the subject and also to Dr. W. O'Reilly for correction of the English and many helpful sug. gestions.

\section{References}

Beyerle, 1931, Archiv fur Elektortechnik, 25, 269.

Bouasse, H., 1914, Cours de Magnétisme et d'électricité, 1. partie, 188.

Clark, J.W., 1938, Rev. Sci. Instr., 9, 320-2.

Daniels, J.M., 1950, Proc. Phys. Soc. London, 63, 1028-34.

Fanselau, V.G., 1929, Z. Phys., 54, 260-9. 
Harris, F.K., 1934-a, J. Res. Nat. Bur. Stand., 12, 87-102.

Harris, F.K., 1934-b, J. Res. Nat. Bur. Stand., 13, 391-410.

Hart, P.J., 1967, Universal Tables for Magnetic Fields of Filamentary and Distributed Circular Currents, Elsevier Pub. Co.

İspir Y., 1966, Rev. Fac. des Sci., Istanbul, 31, 19-23.

Lavaud, J.C., 1964, Thesis, Univ. Paris.

Lyddone, R.H. and Ruark A.E., 1939, Rev. Sci. Instr., 10, 253-7.

Parry, J.H., 1967, Methods in Palaeomagnetism, Elsevier Pub. Co., 551-67.

Rubens, S.M., 1945, Rev. Sci. Instru., 16, 243-5.

Ruark, A.E. and Peters, M.F., 1926, Rev. Sci. Instr., 13, 205-12.

Thellier, E., 1938, Ann. Inst. Phys. Globe, Univ. Paris, 16, 157-302.

Thellier, E., 1967, Methods in Palaemognetism, Elsevier Pub. Co., 149-54. 